\title{
OD stretch vibrational relaxation of HOD in liquid to supercritical $\mathrm{H}_{2} \mathrm{O}$
}

\author{
Tim Schäfer, ${ }^{1}$ Jörg Lindner, ${ }^{2}$ Peter Vöhringer, ${ }^{2}$ and Dirk Schwarzer ${ }^{1, a)}$ \\ ${ }^{1}$ Max-Planck-Institut für biophysikalische Chemie, Am Fassberg 11, D-37077 Göttingen, Germany \\ ${ }^{2}$ Institut für Physikalische und Theoretische Chemie, Universität Bonn, Wegelerstr. 12, \\ D-53115 Bonn, Germany
}

(Received 26 March 2009; accepted 16 May 2009; published online 8 June 2009)

\begin{abstract}
The population relaxation of the OD stretching vibration of HOD diluted in $\mathrm{H}_{2} \mathrm{O}$ is studied by time-resolved infrared pump-probe spectroscopy for temperatures between 278 and $663 \mathrm{~K}$ in the density range $0.28 \leq \rho \leq 1.01 \mathrm{~g} / \mathrm{cm}^{3}$. Transient spectra recorded after exciting the $v=0 \rightarrow 1$ OD stretching transition at low temperatures show a delay between excited state decay and formation of the thermalized spectrum pointing to an intermediately populated state. Above $400 \mathrm{~K}$, the rates of excited state decay and ground state recovery become equivalent and the intermediate state is not detectable anymore. Over the entire thermodynamic range, the derived OD stretch relaxation rate constant $k_{r}$ depends linearly on the static dielectric constant $\varepsilon$ of water, indicating a correlation of $k_{r}$ with the average hydrogen bond connectivity of HOD within the $\mathrm{H}_{2} \mathrm{O}$ network. However, in contrast to the $\mathrm{OH}$ stretch relaxation rate constant of the complementary system of $\mathrm{HOD}$ in $\mathrm{D}_{2} \mathrm{O}$, the low density data of $k_{r}(\varepsilon)$ extrapolate to a nonzero intercept for $\varepsilon \rightarrow 1$. Our analysis suggests that at ambient conditions the OD excited state is mainly depopulated by a direct $v=1 \rightarrow 0$ transition, avoiding the excited $v=1$ HOD bending state. Therefore, at room temperature the detected intermediate is assigned to a nonthermalized state with respect to nuclear degrees of freedom of the solvent molecules, and subsequent formation of the final product spectrum is related to a rearrangement of the hydrogen bond network. Passing over to the gas phase the excited OD stretch state shifts into close resonance with the HOD bend overtone, thereby opening up an additional relaxation channel. (C) 2009 American Institute of Physics. [DOI: 10.1063/1.3151673]
\end{abstract}

\section{INTRODUCTION}

The unusual thermodynamic properties of liquid water can be attributed to its ability to form extended threedimensional hydrogen bonded networks. ${ }^{1}$ These networks are highly dynamic in nature and apart from fluctuations in hydrogen bond lengths and angles are characterized by continuous breaking and reformation of hydrogen bonds on time scales of about 1 ps. $^{2,3}$ In recent years, time-resolved vibrational spectroscopy has proven to be extremely powerful in elucidating the structural dynamics of water. ${ }^{4,5}$ The reason for this is the strong correlation between hydrogen bond strength and the instantaneous $\mathrm{OH}$ stretch vibrator frequency. ${ }^{6-8}$ An important approach is to measure the ultrafast response of the hydrogen bond network induced by vibrational energy relaxation (VER) of selectively excited water stretching ${ }^{-12}$ or bending modes. ${ }^{13-15}$ For the analysis of these experiments it is crucial to know the time scales and pathways of VER in detail. But also in the context of solvation and reaction chemistry in liquid water, energy transport within and between water molecules plays a pivotal role.

For neat $\mathrm{H}_{2} \mathrm{O}$, VER is dominated by intra- and intermolecular resonances resulting in a complete delocalization of an initial vibrational excitation over a large number of neighboring molecules accompanied by an ultrafast memory loss of the transition dipole orientation. ${ }^{16}$ Vibrational delocalization is suppressed in the VER of the $\mathrm{OH}(\mathrm{OD})$ stretching

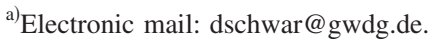

mode of HOD dissolved in $\mathrm{D}_{2} \mathrm{O}\left(\mathrm{H}_{2} \mathrm{O}\right)$. Here isotope substitution lifts inter- and intramolecular resonances, thereby facilitating the observation of the relaxation processes. In the case of $\mathrm{HOD}$ in $\mathrm{D}_{2} \mathrm{O}$, the lifetime of the $\mathrm{OH}$ stretch excited state at ambient conditions was found to be in the range of $0.5-1.0$ ps. $^{9-12,17-23}$ This broad range was attributed to a distribution of hydrogen bonded configurations of the HOD molecule, which on the time scale of VER appears to be partially inhomogeneous. Accordingly, the relaxation is faster for a subensemble of strongly hydrogen bonded $\mathrm{OH}$ oscillators absorbing at the red edge of the steady state $\mathrm{OH}$ stretch band as compared to a weakly hydrogen bonded subensemble excited at the blue edge. ${ }^{22}$ Above $400 \mathrm{~K}$, this dispersion of the $\mathrm{OH}$ stretch lifetime disappears because with increasing temperature the reorganization of the local hydrogen bonded configurations accelerates and, simultaneously, the $\mathrm{OH}$ stretch relaxation becomes slower as the hydrogen bond network is weakened. ${ }^{24,25}$ Interestingly, a linear relation between the $\mathrm{OH}$ stretch relaxation rate constant $k_{r}$ and the static dielectric constant $\varepsilon$ was found. The latter quantity was taken as a measure for the average hydrogen bond connectivity in the bulk fluid. Marked deviations from this linear correlation at low temperatures and, in particular, under ambient conditions were caused by the accidental coincidence of time scales for hydrogen bond reorganization and excited state depopulation. Nevertheless, the linear relation between $k_{r}$ and $\varepsilon$ was used to determine by extrapolation the en- 
semble averaged lifetime of the first excited vibrational quantum state of the $\mathrm{OH}$ stretch mode at ambient conditions to $0.63 \mathrm{ps.}^{24}$

These experimental studies are accompanied by numerous theoretical investigations aimed at elucidating the relaxation pathways in detail. ${ }^{26-30}$ The calculations converge in identifying the transfer from the excited $\mathrm{OH}$ stretch mode to the first overtone of the HOD bend vibration as the rate determining step in the relaxation cascade. ${ }^{26,27}$ Recent nonequilibrium molecular dynamics (MD) simulations suggest a strong coupling of the bending and $\mathrm{OH}$ stretching modes to the HOD rotation which in turn passes the energy to the $\mathrm{D}_{2} \mathrm{O}$ rotation indicating libration-to-libration energy transfer as an important intermolecular relaxation pathway. ${ }^{30}$

The OD stretch relaxation of the complementary system HOD dissolved in $\mathrm{H}_{2} \mathrm{O}$ is more than two times slower ${ }^{31-34}$ than the $\mathrm{OH}$ stretch relaxation of $\mathrm{HOD}$ in $\mathrm{D}_{2} \mathrm{O}$. In contrast to the $\mathrm{OH}$ stretch relaxation, inhomogeneities of the lifetime within the OD stretch absorption band have not been observed at ambient conditions. ${ }^{31}$ This can easily be explained by the longer lifetime of the OD stretch vibration because hydrogen bond dynamics for both systems can be expected to be quite similar. As a result, the experimentally observed decay correspond to an ensemble averaged population relaxation. If this interpretation is correct, the OD stretch relaxation rate constant $k_{r}$ should be strictly proportional to the static dielectric constant of water even down to low temperatures where the marked deviations were previously observed for the $\mathrm{OH}$ stretch relaxation of $\mathrm{HOD}$ in $\mathrm{D}_{2} \mathrm{O}$. To validate this conjecture we performed femtosecond mid-IR pumpprobe spectroscopy on the OD stretching mode of HOD in $\mathrm{H}_{2} \mathrm{O}$ over a broad range of thermodynamic state points from the melting point to beyond the critical temperature of water. By varying the pressure between 1 and 600 bars, the static dielectric constant was adjusted in the range $4.5<\varepsilon<86.3$. Recently, Tielrooij et $a l .{ }^{34}$ observed a $30 \%$ increase in the OD stretch lifetime in the temperature interval $2-70{ }^{\circ} \mathrm{C}$ which, however, is too small to arrive at a meaningful conclusion about the $k_{r}(\varepsilon)$ dependence.

\section{EXPERIMENTAL}

Femtosecond pump-probe measurements were performed with a tunable dual-color midinfrared laser source and frequency-selective detection. A semicommercial Ti:sapphire oscillator/regenerative amplifier system generating 800 $\mathrm{nm}, 100 \mathrm{fs}$ optical pulses with energies of $700 \mu \mathrm{J}$ and a repetition rate of $1 \mathrm{kHz}$ was used to pump two optical parametric amplifiers $^{35,36}$ with a power ratio of 1.5:1. Subsequently, mid-IR pulses at around $2500 \mathrm{~cm}^{-1}$ were obtained from difference frequency mixing of idler and signal of the two optical parametric amplifiers providing pump and probe pulse energies of 4 and $0.5 \mu \mathrm{J}$, respectively. The relative plane of polarization of both pulses was adjusted to $54.7^{\circ}$. The pump pulses were sent through a motorized optical delay line. A portion of the probe pulse was split off and served as a reference which preceded pump and probe pulses by 1.5 ns. Pump, probe, and reference beams were focused into the sample with an off-axis parabolic mirror of $100 \mathrm{~mm}$ focal

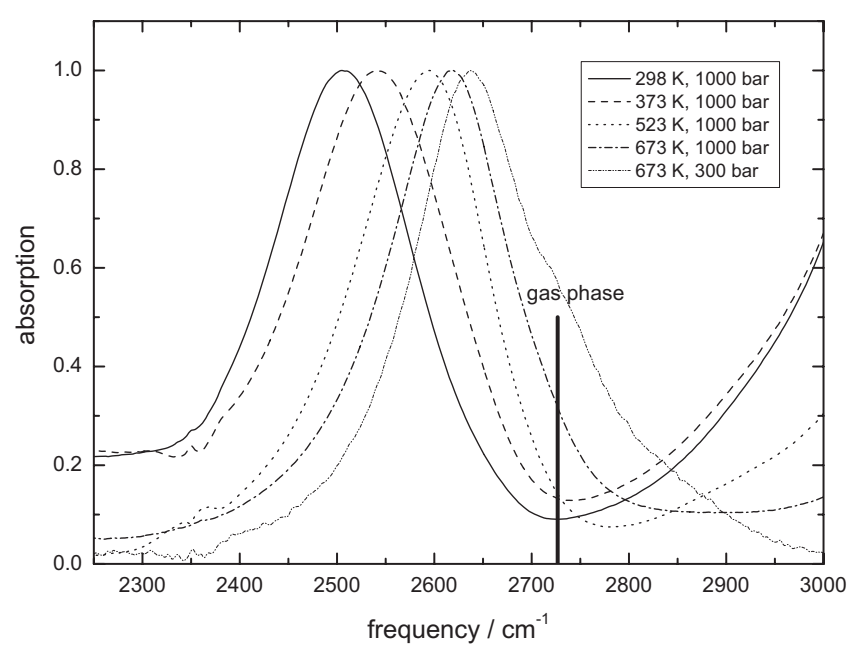

FIG. 1. Linear absorption spectra of $8 \% \mathrm{HOD}$ in $\mathrm{H}_{2} \mathrm{O}$ in the OD stretch region at various thermodynamic state points corresponding to densities of $1.04,1.00,0.877,0.693$, and $0.360 \mathrm{~g} / \mathrm{cm}^{3}$ (left to right).

length. Behind the sample, remaining pump light was captured in a beam dump. Probe and reference beams were fed in a grating polychromator and imaged on a commercial dual row $\mathrm{HgCdTe}$ array detector $(2 \times 32$ elements $)$.

The experiments were performed in a high temperature high-pressure cell capable of operating up to 1500 bars at $750 \mathrm{~K}^{25}$ The cell body was made of a nickel-based high temperature alloy. Sapphire disks of $5 \mathrm{~mm}$ diameter and 2.50 $\mathrm{mm}$ thickness were employed as optical windows. The optical path length inside the cell was adjusted between 30 and $200 \mu \mathrm{m}$. Heating cartridges embedded in the cell body and a thermocouple inside the cell were used to regulate the sample temperature with an accuracy of $\pm 1 \mathrm{~K}$. The pressure was measured with an accuracy of $1 \%$ by a manometer connected to the cell. HOD solutions were prepared by mixing $\mathrm{H}_{2} \mathrm{O}$ with $2 \%-5 \% \mathrm{D}_{2} \mathrm{O}$. For the time-resolved experiments, the sample concentration and optical path length inside the cell were adjusted to produce a peak optical density between 0.5 and 1.0. The density and the dielectric constant for various thermodynamic state points of water were calculated from pressure and temperature data using the PROPATH program package. ${ }^{37}$

\section{RESULTS}

In Fig. 1 normalized infrared spectra of the OD stretching vibration of HOD in water at various thermodynamic state points are shown. With increasing temperature and decreasing density the maximum $\nu_{\max }$ of the OD stretch band shifts from $2507 \mathrm{~cm}^{-1}$ at ambient conditions to $2640 \mathrm{~cm}^{-1}$ at a supercritical temperature of $T=673 \mathrm{~K}$ and a pressure of $p=300$ bars. The gas phase absorption is further blueshifted to $2727 \mathrm{~cm}^{-1}$ as shown by the bar in Fig. 1. These observations are in complete agreement with earlier investigations ${ }^{38}$ and suggest a weakening of the hydrogen bond network as the solvent density is reduced. On the other hand, for the spectrum at lowest density there is still considerable overlap with the room temperature spectrum, indicating the presence of hydrogen bonds even at $673 \mathrm{~K}$ and densities $\rho$ $>0.3 \mathrm{~g} / \mathrm{cm}^{3}$. In analyzing the corresponding $\mathrm{OH}$ stretching 


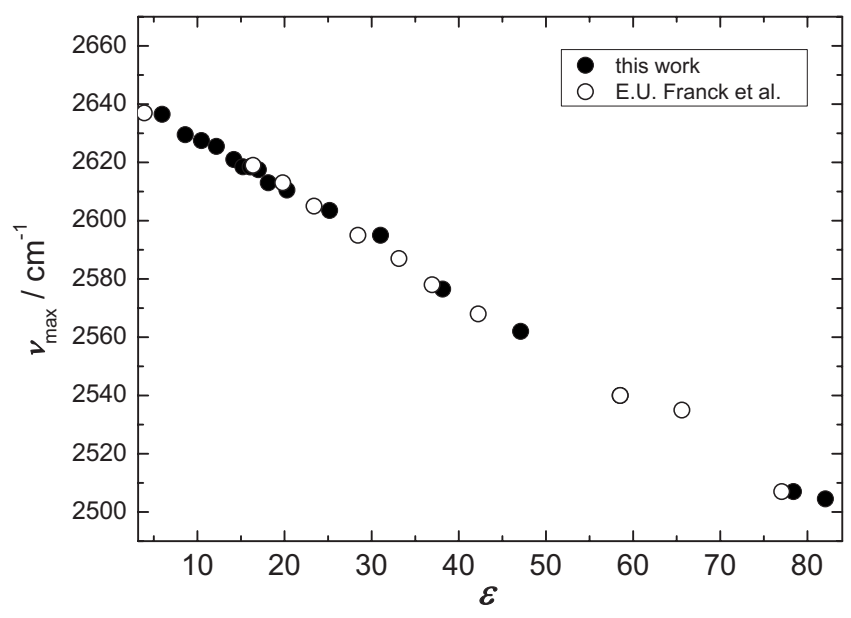

FIG. 2. Maximum of the OD stretch absorption band of HOD vs the static dielectric constant of water. Our data are compared with those of Franck and Roth (Ref. 38).

band of HOD in $\mathrm{D}_{2} \mathrm{O}$ we found a linear dependence of the solvent shift on the static dielectric constant $\varepsilon$ of the solvent. ${ }^{25}$ Figure 2 shows that such a relation also holds for the OD stretching mode.

For the time-resolved experiments, the pump frequency was always adjusted to the center of the OD stretch absorption band at the respective thermodynamic conditions. As an example, results are shown in Figs. 3 and 4 for $\mathrm{HOD}$ in $\mathrm{H}_{2} \mathrm{O}$ at $T=573 \mathrm{~K}$ and $p=300$ bars (corresponding to $\rho$ $=0.75 \mathrm{~g} / \mathrm{cm}^{3}$ ) where the OD stretching vibration $\nu_{\mathrm{OD}}$ was excited at $2600 \mathrm{~cm}^{-1}$. At early pump-probe delay times, the spectra show a pump-induced bleaching/stimulated emission of the $\nu_{\mathrm{OD}}=0 \rightarrow 1$ transition and a corresponding $\nu_{\mathrm{OD}}=1$ $\rightarrow 2$ absorption at frequencies $<2550 \mathrm{~cm}^{-1}$. At later times ( $>26 \mathrm{ps}$ ), a transient spectrum is observed consisting of bleaching at $2550 \mathrm{~cm}^{-1}$ and absorption at $>2670 \mathrm{~cm}^{-1}$, both of which persist for the experimentally accessible time range of $1.5 \mathrm{~ns}$. Similar features have been observed at ambient conditions. $^{32,33}$ The long-time spectrum was attributed to a temperature rise of the sample in the laser focus once the pump energy is transferred from the excited HOD molecule

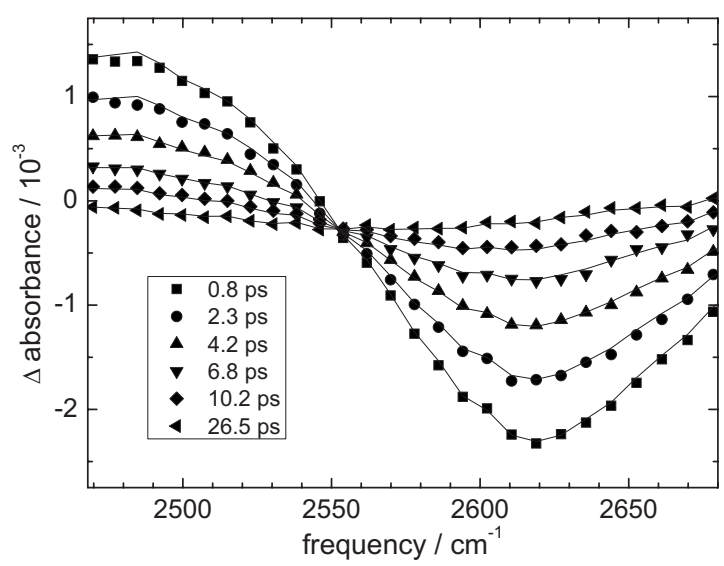

FIG. 3. Transient difference spectra of $6 \% \mathrm{HOD}$ in $\mathrm{H}_{2} \mathrm{O}$ at $573 \mathrm{~K}$ and 300 bars $\left(\rho=0.75 \mathrm{~g} / \mathrm{cm}^{3}\right)$ after excitation with a laser pulse centered at $2600 \mathrm{~cm}^{-1}$. Spectra are shown for different pump-probe delays. The full lines correspond to a fit of a model described in Fig. 5 to the measurements. The derived OD stretch lifetime is $4.6 \mathrm{ps}$.

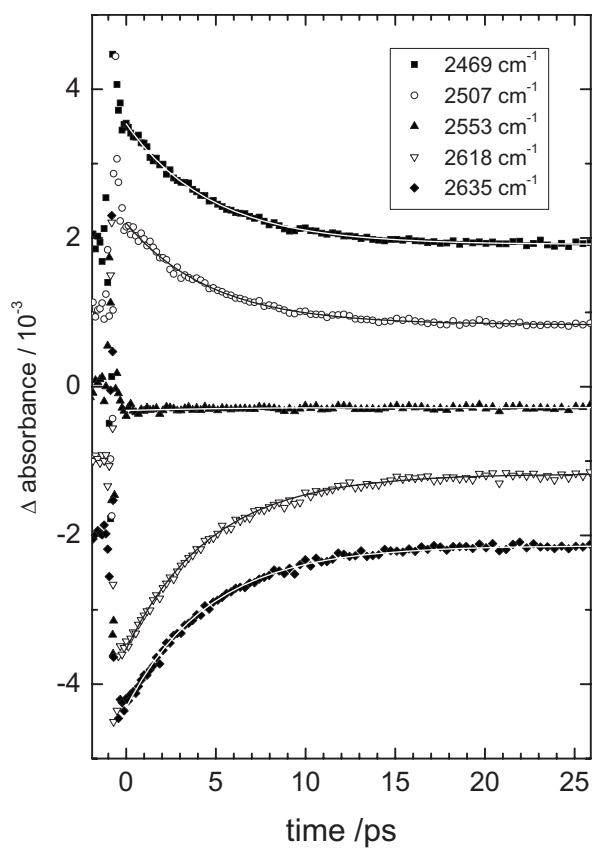

FIG. 4. Time dependent induced absorption signals at various probe frequencies for the same data set as shown in Fig. 3 (for clarity the traces are successively shifted along the vertical axis by a constant amount). The full lines correspond to a fit of a model (Fig. 5) to the data.

to the surrounding water molecules. ${ }^{31,32}$ An increased temperature leads to a blueshift of the spectrum (see Fig. 1), consistent with a long-time differential transmission spectrum that closely resembles a thermal difference spectrum. For the kinetic analysis of our data, we used the same relaxation scheme proposed for room temperature measurements (see Fig. 5). ${ }^{32,33}$ There, it was shown that the appearance of the long-time spectrum involves a significant temporal delay relative to the population decay in $\nu_{\mathrm{OD}}=1$. This finding strongly suggests that an intermediate state $\nu_{\mathrm{OD}}=0^{*}$ of nonthermal character is transiently populated before the initial pump energy can fully dissipate to form a heated laser focal volume.

The nature of this intermediate state is not quite clear. MD calculations on the complementary system HOD in $\mathrm{D}_{2} \mathrm{O}$ suggest $^{26,27,30}$ that after $\mathrm{OH}$ stretching excitation, energy transfer to the HOD bending vibration $\delta_{\mathrm{HOD}}$ takes place. Accordingly, the intermediate state of the $\nu_{\mathrm{OD}}$ relaxation of $\mathrm{HOD}$ in $\mathrm{H}_{2} \mathrm{O}$ was attributed to an excited state of the bending mode. ${ }^{33}$ However, more recent calculations on this sys-

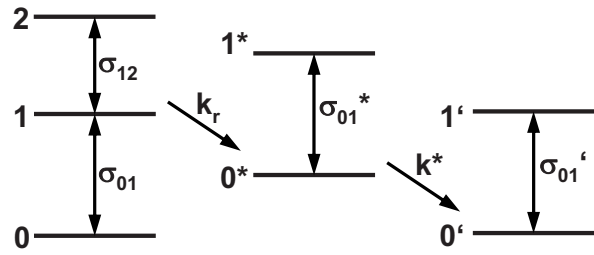

FIG. 5. Kinetic model of the OD stretch relaxation of $\mathrm{HOD}$ in $\mathrm{H}_{2} \mathrm{O}$ (adapted from Ref. 32). After exciting the $\nu_{\mathrm{OD}}=1$ level of the HOD molecule, the population decays to the $\nu_{\mathrm{OD}}=0^{*}$ ground state with a rate constant $k_{r}$. The subsequent thermalization to the $\nu_{\mathrm{OD}}=0^{\prime}$ state is characterized by the rate constant $k^{*}$. The cross sections $\sigma_{01}, \sigma_{01}^{*}$, and $\sigma_{01}^{\prime}$ are identical but depend on the number of molecules reaching the thermalized ground state and, hence, vary with time. The cross section $\sigma_{12}$ is time independent. 


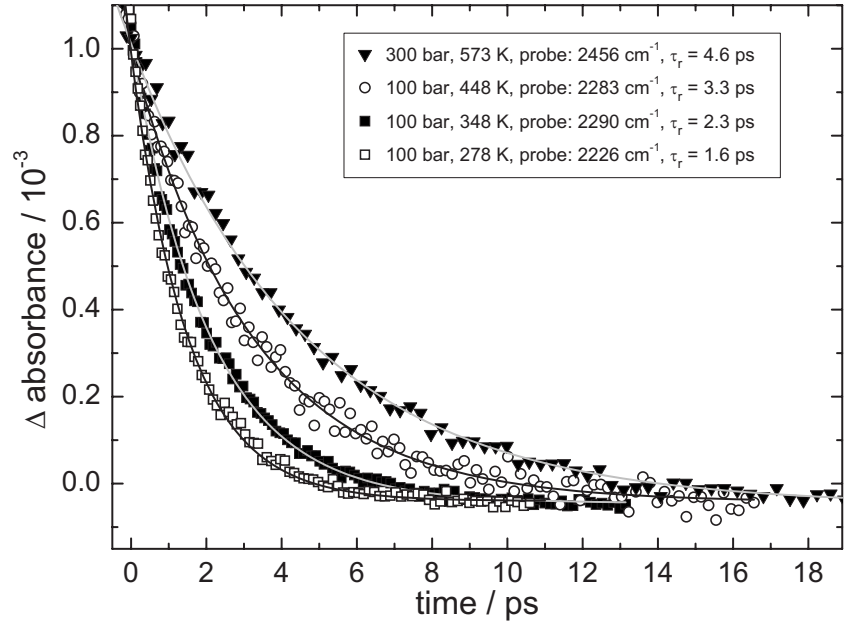

FIG. 6. Normalized transient absorption signals probing the excited state $\nu_{\mathrm{OD}}=1$ of $\mathrm{HOD}$ in $\mathrm{H}_{2} \mathrm{O}$ at various thermodynamic conditions. Full lines correspond to fits of the model presented in Fig. 5 to the data.

tem indicate that the main relaxation channel is the direct $\nu_{\mathrm{OD}}=1 \rightarrow 0$ transition to the ground state, avoiding an excited $\delta_{\text {HOD }}$ state. $^{39}$

Our experiments confirm the existence of an intermediate state, i.e., we can reproduce the room temperature data of, e.g., Refs. 33 and 34 and find $\tau_{r}=1 / k_{r}=1.8$ ps and $\tau^{*}$ $=1 / k^{*}=0.9 \mathrm{ps}$. With increasing temperatures, however, the amplitude of the long-time transient spectrum reminiscent of the heated liquid decreases relative to the transient spectrum at $t=0$. The reason is that the spectral shift of the OD stretch band upon heating is weaker at elevated temperatures than at low temperatures. This can be rationalized by inspecting the temperature dependence of the dielectric constant $\varepsilon$, which in turn is linearly related to the spectral position $\nu_{\max }$ (see Fig. 2). At $298 \mathrm{~K}$ the dielectric constant changes by $0.36 / \mathrm{K}$ whereas at $573 \mathrm{~K}$ it is only $0.12 / \mathrm{K}$, i.e., at the higher temperature the thermal difference spectrum resulting from laser heating is expected to be threefold smaller in amplitude than at ambient conditions. A second point concerns the ratio of $k_{r} / k^{*}$. The time constants derived from our measurements between 278 and $400 \mathrm{~K}$ indicate a twofold increase in $k^{*}$ with temperature whereas $k_{r}$ decreases by almost a factor of 2 (see below) thereby shifting the ratio $k_{r} / k^{*}$ to smaller values. This effect in conjunction with a smaller amplitude of the kinetic component related to $k^{*}$ makes a reliable evaluation of the thermalization rate above $400 \mathrm{~K}$ impossible. On the other hand, it simplifies the evaluation of $k_{r}$ as the temporal response in the mid-IR becomes single exponential and independent of the probe frequency. Consequently, for our $573 \mathrm{~K}$ measurements in Figs. 3 and 4 one can observe an isosbestic point at $2552 \mathrm{~cm}^{-1}$, which is clearly absent at room temperature. ${ }^{33}$

In Fig. 6 transient absorption signals covering a temperature range between 278 and $573 \mathrm{~K}$ are presented. The relaxation time constants derived from fitting the experimental traces by the model of Fig. 5 increase from $1.6 \mathrm{ps}$ at the lowest temperature $\left(\rho=1.00 \mathrm{~g} / \mathrm{cm}^{3}\right)$ to $4.6 \mathrm{ps}$ at the highest temperature $\left(\rho=0.4 \mathrm{~g} / \mathrm{cm}^{3}\right)$. All measured relaxation times
TABLE I. Relaxation times of the OD stretch vibration of $\mathrm{HOD}$ in $\mathrm{H}_{2} \mathrm{O}$.

\begin{tabular}{|c|c|c|c|c|}
\hline $\begin{array}{c}T \\
(\mathrm{~K})\end{array}$ & $\begin{array}{c}p \\
\text { (bar) }\end{array}$ & $\begin{array}{c}\rho^{\mathrm{a}} \\
\left(\mathrm{mol} \mathrm{l^{-1 }}\right)\end{array}$ & $\varepsilon^{\mathrm{a}}$ & $\begin{array}{c}\tau_{r} \\
(\mathrm{ps})\end{array}$ \\
\hline 663 & 260 & 0.285 & 4.5 & $9 \pm 2$ \\
\hline 663 & 290 & 0.45 & 8.4 & $7 \pm 1$ \\
\hline 663 & 330 & 0.51 & 10.2 & $6 \pm 1$ \\
\hline 663 & 390 & 0.56 & 11.7 & $5.9 \pm 0.5$ \\
\hline 663 & 500 & 0.616 & 13.2 & $5.3 \pm 0.5$ \\
\hline 308 & 100 & 1.00 & 75.3 & $1.9 \pm 0.15$ \\
\hline 328 & 100 & 0.99 & 68.6 & $2.2 \pm 0.15$ \\
\hline 348 & 100 & 0.98 & 62.6 & $2.3 \pm 0.15$ \\
\hline 368 & 100 & 0.97 & 57.1 & $2.6 \pm 0.2$ \\
\hline 388 & 100 & 0.95 & 52.1 & $2.6 \pm 0.3$ \\
\hline 408 & 100 & 0.94 & 47.5 & $2.8 \pm 0.3$ \\
\hline 428 & 100 & 0.92 & 43.3 & $3.0 \pm 0.4$ \\
\hline 448 & 100 & 0.90 & 39.5 & $3.3 \pm 0.6$ \\
\hline 468 & 100 & 0.88 & 35.9 & $3.2 \pm 0.7$ \\
\hline 488 & 100 & 0.85 & 32.7 & $3.6 \pm 0.3$ \\
\hline 508 & 100 & 0.83 & 29.6 & $3.8 \pm 0.4$ \\
\hline 528 & 100 & 0.80 & 26.7 & $4.2 \pm 0.6$ \\
\hline 298 & 1 & 1.00 & 78.5 & $1.8 \pm 0.15$ \\
\hline 278 & 100 & 1.01 & 86.3 & $1.6 \pm 0.15$ \\
\hline 293 & 200 & 1.01 & 81.1 & $1.9 \pm 0.15$ \\
\hline 348 & 280 & 0.99 & 63.2 & $2.2 \pm 0.15$ \\
\hline 398 & 500 & 0.96 & 51.0 & $2.6 \pm 0.3$ \\
\hline 423 & 574 & 0.95 & 45.8 & $2.9 \pm 0.5$ \\
\hline 448 & 500 & 0.92 & 40.9 & $3.2 \pm 0.1$ \\
\hline 498 & 500 & 0.87 & 32.8 & $3.6 \pm 0.2$ \\
\hline 548 & 500 & 0.81 & 26.1 & $4.2 \pm 0.3$ \\
\hline 573 & 300 & 0.75 & 21.9 & $4.6 \pm 0.3$ \\
\hline 598 & 475 & 0.74 & 20.1 & $4.8 \pm 0.7$ \\
\hline 613 & 160 & 0.62 & 15.0 & $5.6 \pm 1.2$ \\
\hline 613 & 200 & 0.64 & 15.7 & $5.5 \pm 1.2$ \\
\hline 613 & 211 & 0.64 & 15.9 & $5.3 \pm 0.6$ \\
\hline 643 & 270 & 0.56 & 12.1 & $5.9 \pm 1.2$ \\
\hline
\end{tabular}

${ }^{\mathrm{a}}$ Reference 37.

are summarized in Table I including estimated uncertainties of $\tau_{r}$, which at low temperature are about $10 \%$ and above $T_{c}$ increase to $20 \%$.

\section{DISCUSSION}

In our previous investigation of the complementary system $\mathrm{HOD}$ in $\mathrm{D}_{2} \mathrm{O}$ we have demonstrated that a linear correlation exists between the experimental $\mathrm{OH}$ stretch relaxation rate constant and the temperature and density dependent static dielectric constant $\varepsilon$ of the solvent. ${ }^{24,25}$ Together with MD simulations on a fluctuating charge TIP4P model of water, showing that $\varepsilon$ is proportional to the average number $n_{\mathrm{HB}}$ of hydrogen bonds a water molecule is involved in, ${ }^{40}$ this result could be mapped onto a linear dependence of the relaxation rate constant on $n_{\mathrm{HB}}$. Deviations from this linear relation observed at about room temperature were attributed to H-bond induced spectral diffusion, which appears to occur on a similar time scale as vibrational relaxation under these conditions. If this interpretation is correct, a corresponding plot for the $\mathrm{HOD} / \mathrm{H}_{2} \mathrm{O}$ system investigated here should also yield a linear dependence of the OD stretch relaxation rate constant $k_{r}$ on $\varepsilon$. In contrast to the $\mathrm{HOD} / \mathrm{H}_{2} \mathrm{O}$ system, however, no deviations from this linear correlation are expected 


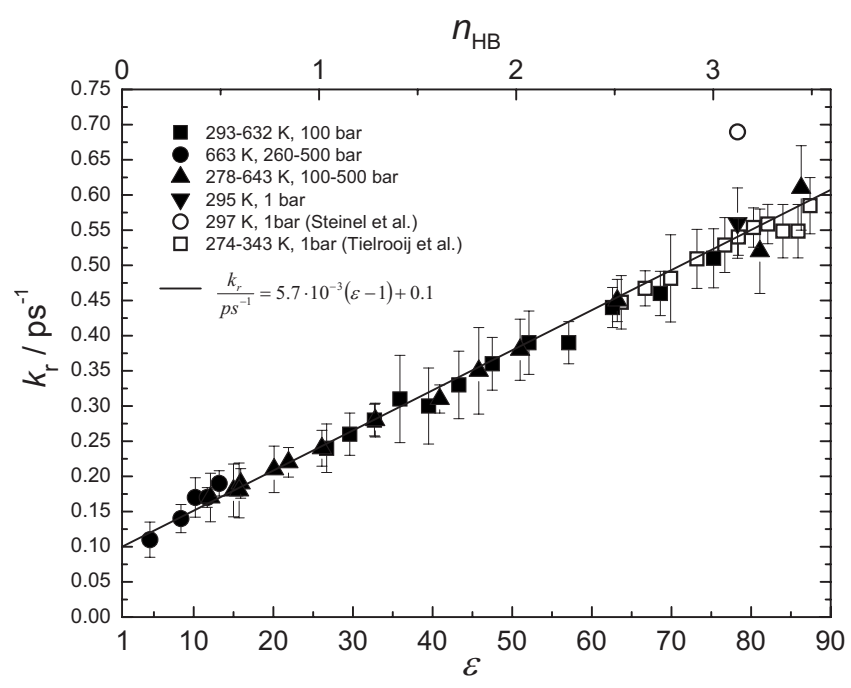

FIG. 7. OD stretch relaxation rate constant of HOD vs the dielectric constant of water. The top axes indicate the average number of hydrogen bonds per water molecule determined from MD calculations (Ref. 40). Open symbols are data from Refs. 34 and 31, respectively. The solid line corresponds to a linear fit to the data points.

at room temperature because here, the OD stretch relaxation rate is known to be 2.5 times slower than the $\mathrm{OH}$ stretch relaxation rate whereas the dynamics of spectral diffusion of $\mathrm{HOD}$ in $\mathrm{D}_{2} \mathrm{O}$ and $\mathrm{H}_{2} \mathrm{O}$ are likely to be similar.

A corresponding plot of $k_{r}$ versus $\varepsilon$ is presented in Fig. 7 combined with a conversion of the dielectric constant to the average number of hydrogen bonds $n_{\mathrm{HB}}$ per water molecule as derived from the MD simulations of Ref. 40. For comparison the data of Bakker and co-workers ${ }^{32-34}$ and Steinel et al. ${ }^{31}$ measured at 1 bar are reproduced by open symbols in Fig. 7. Whereas the agreement with Bakker and co-workers is excellent, the result of Steinel et al. deviates considerably from the rest of the data set. The reason for this discrepancy is most likely due to a different procedure for data analysis used in Ref. 31 (see also Ref. 32 for a discussion of this point).

In general, we cannot rule out that a different model would slightly change our relaxation rate constants for low temperatures. However, as stated above our time traces at $T>400 \mathrm{~K}$ (corresponding to dielectric constants of $\varepsilon<50$ ) are single exponential in nature. Since the extracted time constants are independent of the probe frequency, the relaxation rate constants $k_{r}$ are highly reliable under these conditions. Furthermore, since in Fig. 7 the results from the high temperature data smoothly converge to the low temperature data, we are highly confident that the applied model is adequate to derive correct relaxation times from gas phase all the way up to liquid phase densities.

As in the previous $\mathrm{HOD} / \mathrm{D}_{2} \mathrm{O}$ system, we find within the experimental uncertainty a linear dependence of the OD stretch relaxation rate constant $k_{r}$ on $\varepsilon$ as shown by the solid line in Fig. 7. A closer comparison with the corresponding $\mathrm{OH}$ stretch relaxation rate, however, reveals important differences. First of all, no deviations from the linear relation between $k_{r}$ and $\varepsilon$ can be observed in Fig. 7 at low temperatures (high $\varepsilon$ ). This result supports our conjecture of a clear time scale separation between energy relaxation and $\mathrm{H}$-bond in-

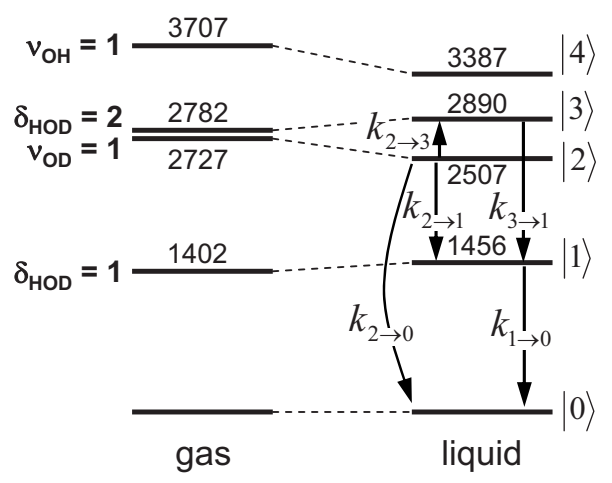

FIG. 8. Vibrational energy levels (in $\mathrm{cm}^{-1}$ ) of HOD in the gas phase (Ref. 41) and in liquid water (Ref. 42) at room temperature.

duced spectral diffusion, i.e. spectral scrambling of the OD stretching vibrational resonance by $\mathrm{H}$-bond dynamics is faster than the OD stretch population dynamics. This holds true from gas phase all the way up to liquid phase densities. Most importantly and in stark contrast to the $\mathrm{HOD} / \mathrm{D}_{2} \mathrm{O},{ }^{24,25}$ this situation also prevails for ambient conditions. Consequently, a single ensemble averaged relaxation rate is always measured in the system HOD in $\mathrm{H}_{2} \mathrm{O}$ with a dependence on neither the excitation nor the probe frequency.

A second difference is related to the high temperature/ low density limit of $k_{r}$. Figure 7 clearly shows that the linear dependence extrapolates to a finite intercept of $k_{r}=0.1 \mathrm{ps}^{-1}$ for $\varepsilon \rightarrow 1$. If we take $\varepsilon$ as a measure of the interaction strength of the excited OD vibration with the surrounding bath, one would expect the rate constant $k_{r}$ to vanish in the limit of the highly diluted gas phase where $\varepsilon$ approaches unity. Note that this asymptotic limit was indeed observed in our previous experiments on the $\mathrm{OH}$ stretch relaxation of $\mathrm{HOD}$ in $\mathrm{D}_{2} \mathrm{O} .^{24,25}$

The offset in Fig. 7 indicates that by changing the thermodynamic conditions continuously from the liquid to the gas phase, a second relaxation channel gradually opens up whose $\varepsilon$ dependence slowly takes over as the density is lowered. The strong influence of the hydrogen bond connectivity on the OD stretch vibrational frequency was already demonstrated in Figs. 1 and 2. As a consequence of these shifts, the relative efficiency of the relaxation pathways will change because the transfer rates critically depend on the energy mismatch between excited and nearby acceptor states. In fact, Fig. 8 shows that upon passing HOD from the liquid to the gas phase, not only the OD stretch mode shifts to higher frequencies but at the same time the bending energy is lowered. Landau-Teller calculations for the liquid environment have identified the direct $\nu_{\mathrm{OD}}=1 \rightarrow 0$ transition as the main pathway of the OD stretch relaxation of $\mathrm{HOD}$ in $\mathrm{H}_{2} \mathrm{O} .{ }^{39}$ Figure 8 suggests the possibility of an additional pathway at low densities involving a transition from the excited OD stretching mode to the bend overtone as both states come into close resonance. The energy mismatch of only $55 \mathrm{~cm}^{-1}$ in the gas phase is below the thermal energy and also below the OD stretch line width, thereby facilitating an efficient stretch-to-bend energy transfer. Once the bend overtone is reached the subsequent relaxation proceeds via $\delta_{\mathrm{HOD}}=1$ to 


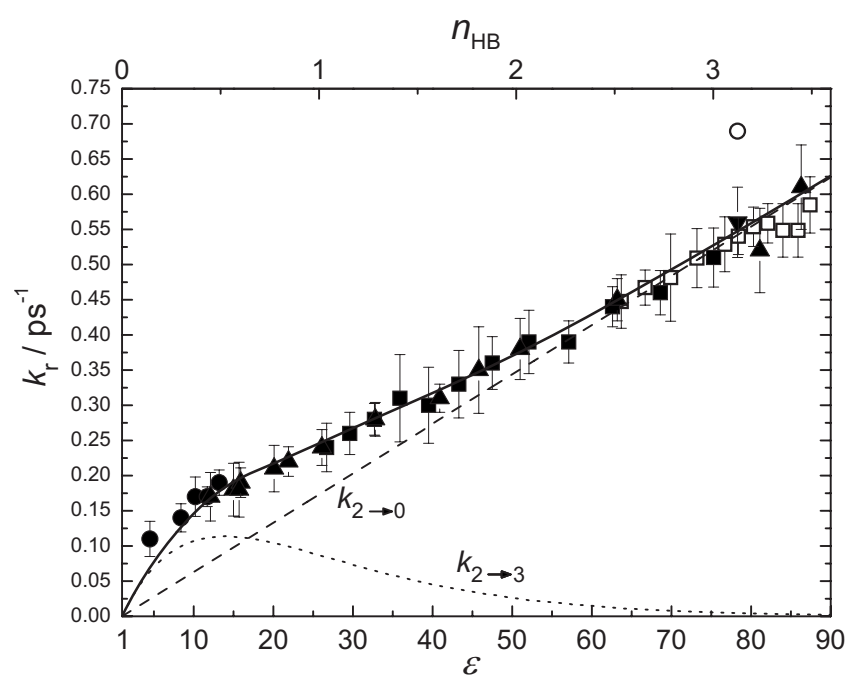

FIG. 9. Comparison between experimental relaxation rate constants and model calculations. The solid line results from model A described in the text and dashed and dotted lines correspond to the individual rate constants of model $\mathrm{A}$ for the direct $\nu_{\mathrm{OD}}=1 \rightarrow 0$ and the $\nu_{\mathrm{OD}}=1 \rightarrow \delta_{\mathrm{HOD}}=2$ transition, respectively.

the ground state. These steps are expected to be relatively fast as in liquid $\mathrm{D}_{2} \mathrm{O}$ the $\delta_{\mathrm{HOD}}=1$ lifetime was found to be only 390 fs. ${ }^{13}$

In the following two kinetic models are elucidated to show that this scenario is able to reproduce the experimental dependence of $k_{r}$ on the dielectric constant in Fig. 7 quantitatively. The two approaches differ in the assignment of the rate determining step that dominates the relaxation in liquid water at room temperature where the pathway via the HOD bend overtone is inaccessible.

Model A follows the suggestion of the recent LandauTeller calculations of $\operatorname{Tian}^{39}$ on the energy transfer under ambient conditions. It assumes that the relaxation mechanism is dominated by the direct transition from $\nu_{\mathrm{OD}}=1$ to $\nu_{\mathrm{OD}}=0$ occurring with the rate coefficient $k_{2 \rightarrow 0}$ (pathway $|2\rangle \rightarrow|0\rangle$ in Fig. 8). In this scenario, the pathway $|2\rangle \rightarrow|1\rangle$ from the $\nu_{\mathrm{OD}}=1$ state to the excited bending vibration is unimportant. In line with our previous studies on the $\mathrm{OH}$ stretching relaxation in $\mathrm{D}_{2} \mathrm{O}$, we regard the dielectric constant as an effective measure of the solute-solvent interaction strength relevant for vibrational relaxation and assume the rate constants $k_{2 \rightarrow 0}$ to be proportional to $\varepsilon-1$ (see also Refs. 24 and 25 for details),

$$
\frac{k_{2 \rightarrow 0}}{\mathrm{ps}^{-1}}=7.0 \times 10^{-3}(\varepsilon-1) .
$$

The prefactor was adjusted to meet the overall relaxation rate $k_{r}$ at room temperature as shown by the dashed line in Fig. 9.

The rate constant $k_{2 \rightarrow 3}$ for the transition $\nu_{\mathrm{OD}}=1$ $\rightarrow \delta_{\mathrm{HOD}}=2$ (state $|2\rangle \rightarrow|3\rangle$ in Fig. 8) is also proportional to the quantity $\varepsilon-1$. However, it carries an additional "energy gap law" dependence to explicitly account for the density dependence of the donor-acceptor energy gap $\Delta E_{23}$ involved in pathway $|2\rangle \rightarrow|3\rangle$ when transiting from gaslike to liquidlike conditions,

$$
k_{2 \rightarrow 3}=a(\varepsilon-1) \exp \left(-\frac{\left|\Delta E_{23}\right|}{c}\right) .
$$

On the basis of the measured solvent shift of the OD stretch band in Fig. 2 we assume that the displacement of all the vibrational states is proportional to $\varepsilon-1$. Hence, one obtains

$$
\frac{\Delta E_{23}}{\mathrm{~cm}^{-1}}=-4.26(\varepsilon-1)-55,
$$

which linearly interpolates the $|2\rangle \rightarrow|3\rangle$ energy gap in Fig. 8 as a function of the dielectric constant between the gas phase $(\varepsilon=1)$ and liquid phase $(\varepsilon=78.4$ at $298 \mathrm{~K})$.

Finally, one has to consider depopulation of the bend vibrational states. For simplicity we again adopt a linear dependence of $k_{3 \rightarrow 1}$ and $k_{1 \rightarrow 0}$ on $\varepsilon-1$. The absolute rates were determined by adjusting $k_{1 \rightarrow 0}$ to the measured relaxation time of the HOD bending vibration in liquid $\mathrm{D}_{2} \mathrm{O}$ at $298 \mathrm{~K}$, ${ }^{13}$

$$
\frac{k_{1 \rightarrow 0}}{\mathrm{ps}^{-1}}=0.034(\varepsilon-1),
$$

and invoking the harmonic approximation within the OD bending manifold, i.e.,

$$
k_{3 \rightarrow 1}=2 k_{1 \rightarrow 0} .
$$

Including detailed balance for all the reverse rate constants,

$$
\frac{k_{\text {up } \rightarrow \text { down }}}{k_{\text {down } \rightarrow \text { up }}}=\exp \left(\frac{\Delta E}{k_{B} T}\right),
$$

we solved the system of kinetic equations resulting from this model numerically and fitted the lifetime of the excited OD stretch vibration to the experimental data using $a$ and $c$ in Eq. (2) as adjustable parameters. The result obtained for the calculated depopulation rate constant with $a=0.065 \mathrm{ps}^{-1}$ and $c=55 \mathrm{~cm}^{-1}$ [Eq. (2)] is shown in Fig. 9 by the full line. To some extend variation of the parameter $c$ can be compensated for by readjusting $a$ without negative effect on the quality of the fit. Accordingly the errors of $c$ and $a$ are $20 \%$ and $50 \%$, respectively. It turns out that within the model, $k_{r}$ is mainly determined by the sum of pathways depopulating the excited OD stretch state,

$$
k_{r}=k_{2 \rightarrow 0}+k_{2 \rightarrow 3},
$$

whereas the reverse processes arising from detailed balance can be neglected. The two individual components $k_{2 \rightarrow 0}$ and $k_{2 \rightarrow 3}$ are presented in Fig. 9 by the dashed and dotted line, respectively.

Considering the simplicity of the model the agreement to the experimental data is excellent. The small value of $c$ $=55 \mathrm{~cm}^{-1}$ indicates a strong dependence of $k_{2 \rightarrow 3}$ on the energy gap. This result is not too surprising because in the Landau-Teller theory, the rate constant for transitions between two anharmonically coupled states $i$ and $f$ depends on the anharmonic transition matrix element $\langle i|q| f\rangle$ and the spectral density of external solvent forces acting on the solute normal mode at the transition frequency $\omega_{i f}$. For small values of $\omega_{i f}$, each of these two quantities shows a strong dependence on the energy gap. ${ }^{26}$ At very low densities (i.e., 
for $\varepsilon<5)$ the model suggests a dependence of $k_{r}$ on $\varepsilon$, which is much stronger than that observed at higher densities. Although we could not measure the dependence in the low- $\varepsilon$ limit directly (because of an insufficient signal to noise ratio at true gas phase densities below $0.2 \mathrm{~g} / \mathrm{cm}^{3}$ ), such a behavior is expected once the transition to the gas phase is completed and the relaxation rate at a given value of $\Delta E_{23}$ is determined by isolated binary collisions.

Model A has a direct impact on the interpretation of the intermediate state $0^{*}$ of Fig. 5. This state was introduced in the analysis of the transient pump-probe spectra because at room temperature the appearance of the thermal difference spectrum due to state $0^{\prime}$ is delayed with respect to the decay of the excited $\nu_{\mathrm{OD}}=1$ population. As excitation of the bending vibration at ambient conditions is avoided, model A excludes the possibility to identify the intermediate $0^{*}$ with the state $\delta_{\mathrm{HOD}}=1$ or any other excited intramolecular vibrational state of HOD. In contrast, $0^{*}$ has to be a nonthermalized state with respect to nuclear degrees of freedom involving motion of the surrounding solvent particles. Rather, the transformation $0^{*} \rightarrow 0^{\prime}$ in Fig. 5 must be related to a rearrangement of the hydrogen bond network after the laser energy is deposited into the sample by vibrational relaxation. ${ }^{31}$

Model B differs from model A by just accounting for efficient population of the excited bending mode at room temperature which permits to assign $0^{*}$ of Fig. 5 to the $\delta_{\mathrm{HOD}}=1$ state. Hence $k_{2 \rightarrow 1}$ in Fig. 8 is assumed to be rate limiting whereas $k_{2 \rightarrow 0}$ is neglected. Similar to Eq. (1) proportionality between $k_{2 \rightarrow 1}$ and the dielectric constant is postulated,

$$
\frac{k_{2 \rightarrow 1}}{\mathrm{ps}^{-1}}=7.0 \times 10^{-3}(\varepsilon-1) .
$$

Equations (2)-(6) of model $\mathrm{A}$ are retained with one modification. In order to attribute the state $0^{*}$ to $\delta_{\mathrm{HOD}}=1$, the bend relaxation rate constant has to be given by

$$
\frac{k_{1 \rightarrow 0}}{\mathrm{ps}^{-1}}=0.014(\varepsilon-1),
$$

which ascertains agreement with the experimentally observed lifetime of $\tau^{*}=0.9 \mathrm{ps}$ at ambient conditions. Choosing $a=0.080 \mathrm{ps}^{-1}$ and $c=55 \mathrm{~cm}^{-1}$ [in Eq. (2)] model B produces an almost identical $k_{r}(\varepsilon)$ dependence as model A (see the full line in Fig. 9).

To decide whether the OD stretch relaxation of HOD in $\mathrm{H}_{2} \mathrm{O}$ follows model $\mathrm{A}$ or $\mathrm{B}$ one has to discuss the impact of elevated temperatures on the nature of the intermediate state attributed to $0^{*}$. For temperatures lower than $400 \mathrm{~K}(\varepsilon$ $>50$ ), relaxation through the bending overtone $\delta_{\mathrm{HOD}}=2$ is negligible compared to pathways populating the lower lying vibrational states regardless of the model. According to model $\mathrm{B}$, the state $0^{*}$ is assigned to the first excited state of the bending mode and therefore the apparent rate coefficients $k_{r}$ and $k^{*}$ are equal to the rate constants $k_{2 \rightarrow 1}$ and $k_{1 \rightarrow 0}$, respectively. Since these two rate constants depend linearly on $\varepsilon-1$, one would expect the ratio of the apparent rates $k_{r} / k^{*}$ to remain constant upon heating. Likewise, the relative amplitudes of the two corresponding kinetic components should be invariant to temperature variations. Neither conclusion can be confirmed by our experiments. Quite in contrast, we observe that the ratio $k_{r} / k^{*}$ as well as the amplitude of the kinetic component related to $k^{*}$ decrease when the temperature is raised. Indeed, above $400 \mathrm{~K}$ the thermalization rate constant cannot be determined reliably any more.

These findings are in much better agreement with the scenario outlined in model A where $k^{*}$ corresponds to a delayed response of the hydrogen bond network upon energy absorption of the $\mathrm{H}_{2} \mathrm{O}$ solvent following OD stretch vibrational relaxation. As was suggested previously, ${ }^{31}$ the time constant of $\tau^{*}=0.9 \mathrm{ps}$ at room temperature is completely consistent with the excitation of low frequency intra- and intermolecular solvent modes which subsequently leads to a breaking of hydrogen bonds. Note that in neat liquid water, a very similar time constant was also attributed to the dynamics of hydrogen bond rearrangement concurrent with a local heating of the pump focal volume. ${ }^{14,15}$ With increasing temperature this process is expected to speed up relative to OD stretch energy relaxation and at a certain point cannot be detected anymore. As a result the measured spectrotemporal response becomes much simpler and isosbestic points such as that shown in Fig. 3 for $T=573 \mathrm{~K}$ can be detected.

\section{CONCLUSIONS}

We have performed femtosecond midinfrared pumpprobe spectroscopy to explore the dynamics of VER of the OD stretching mode of $\mathrm{HOD}$ in $\mathrm{H}_{2} \mathrm{O}$ for elevated pressures and temperatures ranging into the supercritical phase. The measured OD stretch lifetimes vary between $1.6 \mathrm{ps}$ in liquid water at $278 \mathrm{~K}$ and $9 \mathrm{ps}$ in the supercritical state at $663 \mathrm{~K}$ and 260 bars. Similar to the previously measured $\mathrm{OH}$ stretch relaxation of $\mathrm{HOD}$ in $\mathrm{D}_{2} \mathrm{O}$, we observe a linear dependence of the OD stretch relaxation rate constant $k_{r}$ on the static dielectric constant $\varepsilon$ of water. Using the results of MD simulations $^{40}$ this finding implicates an immediate correlation between the relaxation rate and the average number of hydrogen bonds in which the HOD molecule is engaged. In contrast to the $\mathrm{HOD} / \mathrm{D}_{2} \mathrm{O}$ system, however, no deviation from linearity can be observed at low temperatures. The reason for this apparently simpler behavior lies in a clear time scale separation that persists from gas phase to liquid phase densities between the relatively slow OD stretch population relaxation and the faster hydrogen bond dynamics. A second difference concerns the extrapolated low density limit of $k_{r}$, which for the accessible $\varepsilon$ range yields a finite intercept whereas for the $\mathrm{OH}$ stretch relaxation of $\mathrm{HOD}$ in $\mathrm{D}_{2} \mathrm{O}$ it vanishes. Our analysis suggests that level shifting causes an alteration of the prevailing relaxation mechanism as the thermodynamic conditions are changed from the liquid to gas phase. In accordance with recent Landau-Teller calculations ${ }^{39}$ the dominant relaxation pathway in liquid water at room temperature is the direct $v=1 \rightarrow 0$ transition of the OD stretch excited state. The energy is transferred into nuclear degrees of freedom of the solvent leading to a rearrangement of the hydrogen bond network within 0.9 ps. In the gas phase, the energy of the excited OD stretch state is only $55 \mathrm{~cm}^{-1}$ below the HOD bend overtone which opens up 
an efficient relaxation channel involving the cascade $\nu_{\mathrm{OD}}$ $=1 \rightarrow \delta_{\mathrm{HOD}}=2 \rightarrow \delta_{\mathrm{HOD}}=1 \rightarrow$ ground state, where the first step is rate limiting.

\section{ACKNOWLEDGMENTS}

The authors wish to thank Professor Jürgen Troe for continued support. Financial support of this work by the Fonds der Chemischen Industrie and by the Deutsche Forschungsgemeinschaft (Grant NO. VO 593/6-1) is gratefully acknowledged.

${ }^{1}$ Water: A Comprehensive Treatise, edited by F. Franks (Plenum, New York, 1972), Vol. 1.

${ }^{2}$ R. Rey, K. B. Moller, and J. T. Hynes, J. Phys. Chem. A 106, 11993 (2002).

${ }^{3}$ C. P. Lawrence and J. L. Skinner, J. Chem. Phys. 118, 264 (2003).

${ }^{4}$ Ultrafast Hydrogen Bonding Dynamics and Proton Transfer Processes in the Condensed Phase, edited by T. Elsaesser and H. J. Bakker (Kluwer, Dordrecht, 2002).

${ }^{5}$ E. T. J. Nibbering and T. Elsaesser, Chem. Rev. (Washington, D.C.) 104, 1887 (2004)

${ }^{6}$ A. Novak, Struct. Bonding (Berlin) 18, 177 (1974).

${ }^{7}$ W. Mikenda, J. Mol. Struct. 147, 1 (1986).

${ }^{8}$ A. Kandratsenka, D. Schwarzer, and P. Vöhringer, J. Chem. Phys. 128 244510 (2008).

${ }^{9}$ R. Laenen, C. Rauscher, and A. Laubereau, Phys. Rev. Lett. 80, 2622 (1998).

${ }^{10}$ S. Woutersen, U. Emmerichs, H.-K. Nienhuys, and H. J. Bakker, Phys. Rev. Lett. 81, 1106 (1998).

${ }^{11}$ G. M. Gale, G. Gallot, and N. Lascoux, Chem. Phys. Lett. 311, 123 (1999).

${ }^{12}$ A. J. Lock and H. J. Bakker, J. Chem. Phys. 117, 1708 (2002).

${ }^{13}$ P. Bodis, O. F. A. Larsen, and S. Woutersen, J. Phys. Chem. A 109, 5303 (2005)

${ }^{14}$ S. Ashihara, N. Huse, A. Espagne, E. T. J. Nibbering, and T. Elsaesser, J. Phys. Chem. A 111, 743 (2007).

${ }^{15}$ J. Lindner, D. Cringus, M. S. Pshenichnikov, and P. Vöhringer, Chem. Phys. 341, 326 (2007).

${ }^{16}$ M. L. Cowan, B. D. Bruner, N. Huse, J. R. Dwyer, B. Chugh, E. T. J. Nibbering, T. Elsaesser, and R. J. D. Miller, Nature (London) 434, 199
(2005).

${ }^{17}$ S. Woutersen, U. Emmerichs, and H. J. Bakker, Science 278, 658 (1997).

${ }^{18}$ S. Bratos, G. M. Gale, G. Gallot, F. Hache, N. Lascoux, and J.-C. Leicknam, Phys. Rev. E 61, 5211 (2000).

${ }^{19}$ G. M. Gale, G. Gallot, F. Hache, N. Lascoux, S. Bratos, and J.-C. Leicknam, Phys. Rev. Lett. 82, 1068 (1999).

${ }^{20}$ H.-K. Nienhuys, S. Woutersen, R. A. van Santen, and H. J. Bakker, J. Chem. Phys. 111, 1494 (1999).

${ }^{21}$ R. Laenen, C. Rauscher, and A. Laubereau, J. Phys. Chem. B 102, 9304 (1998).

${ }^{22}$ Z. Wang, A. Pakoulev, Y. Pang, and D. D. Dlott, J. Phys. Chem. A 108, 9054 (2004)

${ }^{23}$ Z. Wang, Y. Pang, and D. D. Dlott, Chem. Phys. Lett. 397, 40 (2004).

${ }^{24}$ D. Schwarzer, J. Lindner, and P. Vöhringer, J. Chem. Phys. 123, 161105 (2005).

${ }^{25}$ D. Schwarzer, J. Lindner, and P. Vöhringer, J. Phys. Chem. A 110, 2858 (2006).

${ }^{26}$ R. Rey and J. T. Hynes, J. Chem. Phys. 104, 2356 (1996).

${ }^{27}$ C. P. Lawrence and J. L. Skinner, J. Chem. Phys. 119, 1623 (2003).

${ }^{28}$ C. P. Lawrence and J. L. Skinner, J. Chem. Phys. 119, 3840 (2003).

${ }^{29}$ R. Rey, K. B. Moller, and J. T. Hynes, Chem. Rev. (Washington, D.C.) 104, 1915 (2004).

${ }^{30}$ A. Kandratsenka, J. Schroeder, D. Schwarzer, and V. S. Vikhrenko, J. Chem. Phys. 130, 174507 (2009).

${ }^{31}$ T. Steinel, J. B. Ashbury, J. Zheng, and M. D. Fayer, J. Phys. Chem. A 108, 10957 (2004)

${ }^{32}$ Y. L. A. Rezus and H. J. Bakker, J. Chem. Phys. 123, 114502 (2005).

${ }^{33}$ H. J. Bakker, Y. L. A. Rezus, and R. L. A. Timmer, J. Phys. Chem. A 112, 11523 (2008)

${ }^{34}$ K. J. Tielrooij, C. Petersen, Y. L. A. Rezus, and H. J. Bakker, Chem. Phys. Lett. 471, 71 (2009).

${ }^{35}$ R. A. Kaindl, M. Wurm, K. Reimann, P. Hamm, A. M. Weiner, and M. Woerner, J. Opt. Soc. Am. B 17, 2086 (2000).

${ }^{36}$ P. Hamm, R. A. Kaindl, and J. Stenger, Opt. Lett. 25, 1798 (2000)

${ }^{37}$ PROPATHGroup, PROPATH, a program package for thermophysical properties, Version 12.1, 2001, Web site http://gibbs.mech.kyushu-u.ac.jp.

${ }^{38}$ E. U. Franck and K. Roth, Faraday Discuss. Chem. Soc. 43, 108 (1967).

${ }^{39}$ G. Tian, Chem. Phys. 328, 216 (2006).

${ }^{40}$ N. Yoshii, S. Miura, and S. Okazaki, Chem. Phys. Lett. 345, 195 (2001).

${ }^{41}$ W. S. Benedict, H. Gailar, and E. K. Plyler, J. Chem. Phys. 24, 1139 (1956).

${ }^{42}$ M. Falk and T. A. Ford, Can. J. Chem. 44, 1699 (1966). 